\title{
Tumorigenesis induced by the HHV8-encoded chemokine receptor requires ligand modulation of high constitutive activity
}

\author{
Peter J. Holst, ${ }^{1,2}$ Mette M. Rosenkilde, ${ }^{1}$ Denise Manfra, ${ }^{2}$ Shu-Cheng Chen, ${ }^{2}$ \\ Maria T. Wiekowski, ${ }^{2}$ Birgitte Holst, ${ }^{1}$ Felix Cifire, ${ }^{3}$ Martin Lipp, ${ }^{3}$ Thue W. Schwartz, ${ }^{1}$ \\ and Sergio A. Lira ${ }^{2}$ \\ ${ }^{1}$ Laboratory for Molecular Pharmacology, Department of Pharmacology, Panum Institute, University of Copenhagen, \\ Copenhagen, Denmark \\ ${ }^{2}$ Department of Immunology, Schering-Plough Research Institute, Kenilworth, New Jersey, USA \\ ${ }^{3}$ Max-Delbruck-Center for Molecular Medicine, Berlin, Germany \\ Address correspondence to: Sergio A. Lira, Department of Immunology, Schering-Plough Research Institute, \\ 2015 Galloping Hill Road, Kenilworth, New Jersey 07033, USA. \\ Phone: (908) 740-3088; Fax: (908) 740-3084; E-mail: sergio.lira@spcorp.com.
}

Received for publication June 26, 2001, and accepted in revised form October 22, 2001.

ORF74 (or KSHV-vGPCR) is a highly constitutively active G protein-coupled receptor encoded by HHV8 that is regulated both positively and negatively by endogenous chemokines. When expressed in transgenic mice, this chemokine receptor induces an angioproliferative disease closely resembling Kaposi sarcoma (KS). Here we demonstrate that several lines of mice carrying mutated receptors deficient in either constitutive activity or chemokine regulation fail to develop KS-like disease. In addition, animals expressing a receptor that preserves chemokine binding and constitutive activity but that does not respond to agonist stimulation have a much lower incidence of angiogenic lesions and tumors. These results indicate that induction of the KS-like disease in transgenic mice by ORF74 requires not only high constitutive signaling activity but also modulation of this activity by endogenous chemokines.

J. Clin. Invest. 108:1789-1796 (2001). DOI:10.1172/JCI200113622.

\section{Introduction}

Kaposi sarcoma (KS) is an angioproliferative disease characterized by proliferation of mesenchymal cells with formation of vascular spaces surrounded by spindle-shaped cells and inflammatory cells. Evidence for an infectious etiology has accumulated since the discovery of a novel herpesvirus within KS lesions, called human herpesvirus 8 (HHV8) or KS-associated herpesvirus (KSHV) (1). Infection with HHV8 has been shown to precede development of KS (2) and expression of HHV8-encoded genes has been detected in KS lesions (3). HHV8 encodes various genes with possible transforming or angiogenic properties, including viral homologues of cyclin-D, IL-6, interferon regulatory factors, BCL-2, FLICE-inhibitory protein, three chemokines (vMip-I, vMip-II, and vMip-III), and a G protein-coupled receptor (referred to as $\mathrm{KSHV}-$ vGPCR or ORF74) (4). Expression of ORF74 in transfected cells has been shown to mediate cell proliferation and induce the secretion of VEGF, a potent angiogenic factor expressed within KS lesions (5). It has been suggested that ORF74-expressing cells could contribute to the pathogenesis of KS, possibly through paracrine mechanisms $(5,6)$. This hypothesis was strongly supported by our recent observation that transgenic mice expressing ORF74 under the control of the human CD2 promoter develop angiogenic lesions that have a striking resemblance to human KS lesions, thereby implicating this virally encoded chemokine receptor in the pathogenesis of KS (7).

ORF74 signals constitutively through a phospholipase $\mathrm{C}$ pathway leading to increased phosphatidylinositol turnover and activation of c-Jun N-terminal kinase and p38 mitogen-activated protein kinase (8). The constitutive activity of ORF74 is regulated by a broad spectrum of CXC chemokines that bind this receptor with high affinity. These chemokines can act as agonists, increasing the constitutive signaling of the receptor; neutral ligands, with no impact on signaling; or, as inverse agonists, decreasing the high constitutive signaling activity $(9,10)$. Interestingly, ligands that act as agonists on this virally encoded receptor, are angiogenic chemokines such as GRO $\alpha$, whereas the ligands that act as inverse agonists are angiostatic chemokines such as IP-10 and SDF- $1 \alpha$ $(10,11)$. The CXC chemokines IL-8 and ENA78, which also bind with high affinity to ORF74, are neutral ligands that do not affect the signaling of the receptor. Because KS lesions are highly vascularized and because the signaling activity of ORF74 appears to be regulated by angiogenic and angiostatic chemokines, it could be suggested that fine-tuning of the constitutive activity of ORF74 may be important for the angiogenic and tumorigenic properties of the receptor.

We have previously generated mutant forms of ORF74 in which properties such as constitutive activity, chemokine binding, and agonist activity were selec- 
tively eliminated (12). In the present study we describe the generation and analysis of a number of transgenic lines expressing these ORF74 mutants. We demonstrate that not only the high constitutive signaling activity of ORF74, but also the modulation of this activity by endogenous chemokines, are required for the development of the KS-like disease in transgenic mice.

\section{Methods}

Mice. Transgenic mice expressing HHV8 ORF74 have been described by Yang et al. (7), and the construction of mutated forms of HHV8 ORF74 have been described by Rosenkilde et al. (12). Briefly, the wild-type and mutant forms of ORF74 described by Rosenkilde et al. (12) (Figure 1) were cloned into a hCD2 promoter-driven vector (7) and their sequence verified using an Alfexpress sequencer (Amersham Pharmacia Biotech, Piscataway, New Jersey, USA). Generation of transgenic founders was done as described previously by pronuclear injection of $\mathrm{C} 57 \mathrm{Bl} / 6 \mathrm{~J} \times \mathrm{DBA} / \mathrm{J} \mathrm{F} \mathrm{F}_{2}$ eggs (7). Transgenic animals (L91D, $\Delta 22$, CDVG, R2H) were mated to $\mathrm{C} 57 \mathrm{Bl} / 6 \mathrm{~J} \times \mathrm{DBA} / \mathrm{J} \mathrm{F}_{1}$. Transgenic offspring were identified by PCR amplification of a segment of the ORF74 gene using the following primers: $5^{\prime}$-TGGAACGTTGGAATACTCTCTCTG-3' and 5'-AGGTACCTCACTAGACTGACGCAC- $3^{\prime}$. As an internal control for the amplification, reaction primers for the endogenous LDL gene were used (5'-ACCCAAGACGTGCTCCCAGGATGA3'; $5^{\prime}$ CGCAGTGCTCCTCATCTGACTTGT-3'). These primers amplify a 250-bp segment of the transgene and a 397-bp segment of the LDL gene. Animals were kept under specific pathogen-free conditions, and experiments followed institutional guidelines.

IP3 turnover. COS-7 cells were grown at $10 \% \mathrm{CO}_{2}$ at $37^{\circ} \mathrm{C}$ in DMEM 1885 with $10 \%$ FCS, 2 mM glutamine, and $0.01 \mathrm{mg} / \mathrm{ml}$ gentamycin added. Cells $\left(5 \times 10^{5}\right.$ per well) were transfected with $40 \mu \mathrm{g}$ of plasmid DNA using the calcium precipitation method. One day after transfection the cells were incubated with $5 \mu \mathrm{Ci}$ of ${ }^{3} \mathrm{H}$ myo-inositol per milliliter of inositol-free media for 24 hours. Cells were washed twice in HEPES buffer supplemented with $140 \mathrm{mM} \mathrm{NaCl}, 5 \mathrm{mM} \mathrm{KCl}, 1 \mathrm{mM}$ $\mathrm{MgSO}_{4}, 1 \mathrm{mM} \mathrm{CaCl} 2,10 \mathrm{mM}$ glucose, and $0.05 \%$ (wt/vol) BSA. Cells were then incubated for 90 minutes in HEPES buffer supplemented with $10 \mathrm{mM} \mathrm{LiCl}$ in the presence of chemokines. Cells were extracted with $10 \%$ perchloric acid and incubated on ice for $30 \mathrm{~min}$ utes. Supernatants were neutralized with $\mathrm{KOH}$ in HEPES buffer. ${ }^{3} \mathrm{H}$-inositol phosphates were purified on AG 1-x8 anion-exchange resin (13). Determinations were made in duplicate.

$R N A$ analysis. Thymuses or lesional tissues were dissected, frozen in liquid nitrogen or on dry ice, and RNA isolated using Tri-Reagent (Molecular Research Center Inc., Cincinnati, Ohio, USA) as instructed by the manufacturer, except for an additional extraction step in TriReagent. For transgene expression analysis $15 \mu \mathrm{g}$ of total thymus RNA was run on a $1 \%$ agarose gel and blotted onto a Genescreen membrane (NEN Life Science
Products Inc., Boston, Massachusetts, USA). A 1-kb PCR fragment of the HHV8 ORF74 DNA was labeled with ${ }^{32} \mathrm{P}$ (Amersham Pharmacia Biotech) and used as a probe. For detection of chemokine and ORF74 expression within control and lesional tissue cDNA was synthesized from $2 \mu \mathrm{g}$ total RNA primed with oligo(dT)12-18 using the Superscript II Preamplification System (Invitrogen Corp., Carlsbad, California, USA) as described by the manufacturer. Five microliters of cDNA ( $2 \mu \mathrm{l}$ for GAPDH) was amplified in a $50-\mu \mathrm{l}$ reaction volume using the following conditions: $94^{\circ} \mathrm{C}$ for 1 minute, then 35 cycles at $94^{\circ} \mathrm{C}$ for 1 minute, $60^{\circ} \mathrm{C}$ for 1 minute, $72^{\circ} \mathrm{C}$ for 2 minutes, incubated in a gene Amp PCR System (Applied Biosystems, Foster City, California, USA). The oligonucleotide primers corresponding to the sense and antisense strands, respectively, were as follows: ORF74, 5'-ATGGCGGCCGAGGATTTCCTAACC$3^{\prime}$ and $5^{\prime}$-AGGTACCTCACTAGACT-GACGCAC-3'; KC, $5^{\prime}$ GTCCCGCTGACCAAGAGTCTT- $3^{\prime}$ and $5^{\prime}$-GGCACTGACAGCGCAGCT-3'; SDF-1 $\alpha, 5^{\prime}$-CGTGAGGCCAGGGAAGAGT- $3^{\prime}$ and $5^{\prime}$-TGATGAGCATGGTG-GGTTGA- $3^{\prime}$; MIP2, 5'-CCTGCCAAG-GGTTGACTTCA-3' and $5^{\prime}$-TTCTGTCTGGGCGCAGTG-3'; IP-10， 5'-GACGGTCCGCTGCAACTG- $3^{\prime}$ and $5^{\prime}$-GCTTCCCTATGGCCCTCATT- $3^{\prime}$; and GAPDH, 5'-TGAAGGTCGGTGTGA ACGGATTTGGC-3' and $5^{\prime}$-CATGTAAGGCCATGAGGTCCACCAC- 3 '. The final PCR products were analyzed on a $2 \%$ agarose gel containing ethidium bromide.

Flow cytometry. Single cell suspensions were prepared from the thymus by passage through a $100-\mu \mathrm{M}$ nylon cell strainer (Becton Dickinson Labware, Franklin Lakes, New Jersey, USA) in RPMI medium containing $10 \%$ FCS. Cells $\left(10^{5}\right.$ to $\left.10^{6}\right)$ were incubated with $3 \%$ heat-inactivated mouse serum (Sigma Chemical Co., St. Louis, Missouri, USA) in $1 \times$ PBS, $1 \%$ BSA, and $0.1 \%$ sodium azide for 10 minutes at $4^{\circ} \mathrm{C}$. The cells were stained with a rat $\mathrm{mAb}$ specific for the $\mathrm{N}$-terminus of ORF74 (clone 7D1). This mAb was made according to methods described recently (14) and its generation will be reported elsewhere (F. Cifire and M. Lipp, unpublished observations). Thymocytes expressing ORF74 were detected using mouse anti-rat IgG-FITC Ab (212096-102; Jackson ImmunoResearch Laboratories Inc., West Grove, Pennsylvania, USA) at $0.2 \mathrm{mg} / \mathrm{ml}$ final concentration. To determine viability, samples were subsequently stained with $20 \mu \mathrm{lof} 5 \mu \mathrm{g} / \mathrm{ml}$ propidium iodide (Calbiochem-Novabiochem International, San Diego, California, USA). Events were acquired on a Becton Dickinson FACSCalibur (Becton Dickinson Immunocytometry Systems, Mansfield, Massachusetts, USA) and analyzed using the CellQuest software.

Histology. Fresh mouse tissues were fixed in 10\% phosphate-buffered formalin and processed for paraffin sections. Paraffin sections $(5 \mu \mathrm{m})$ were prepared and stained with hematoxylin and eosin (H\&E).

Statistical analysis. The significance of angioproliferative disease incidence between different transgenic animals was determined by likelihood comparisons, comparing homogeneity across groups (null hypothesis) 
with intergroup heterogeneity, given the following stipulations. (a) The groups are represented by grouped founders and lines of CDVG, R2H, 222 , and L91D animals. (b) The binomial probability of disease development varies between lines in different transgenes. (c) Disease incidence within lines falls in three risk classes with disease probability values of 0 , intermediate, and 1. Thus the null (alternative) hypothesis claims that the four transgenic categories have the same potential to develop angioproliferative disease as reflected in the mix of the three risk classes and the magnitude of the intermediate risk involved.

\section{Results}

ORF74 is responsive to endogenous mouse chemokines. ORF74 binds a number of human CXC chemokines $(6,9,10)$, and, as demonstrated previously, is constitutively active. In vitro experiments have shown that phosphoinositol (PI) turnover levels observed in cells transfected with ORF74 are 5.4-fold above control cells transfected with an empty expression vector (12). To study the biological activities of mutant forms of this receptor in transgenic mice we first determined the ability of the wild-type ORF74 to react with the corresponding mouse chemokine ligands in vitro. To study the interaction of murine chemokines with the wild-type receptor we focused on murine homologues of chemokines known to alter the signaling of ORF74. Thus, initially we used the murine CXC chemokines, MIP-2 and $\mathrm{KC}$, which are most closely related to the preferred ORF74 agonist,
GRO $\alpha$. MIP-2 stimulated inositol phosphate turnover in COS-7 cells transiently transfected with the wild-type ORF-74 to the same degree as human GRO $\alpha$ (Figure 1b). In contrast, the murine peptide $\mathrm{KC}$ had no effect in this system. Both murine IP-10 and murine SDF- $1 \alpha$ acted as inverse agonists in a manner similar to their human counterparts (Figure 1b).

We next examined the ability of the murine chemokines to affect different ORF74 mutants (Figure 1a). Deletion of amino acids in the amino-terminal region of ORF74 has been shown previously to eliminate the ability of human chemokines to affect PI turnover $(12,15)$. One such mutant $(\Delta 22$-ORF74) has been shown to have high constitutive activity and to not respond to chemokines (12). Here we show that none of the murine chemokines tested affected the high constitutive activity of the $\Delta 22$-ORF74 mutant (Figure 1b). The [R208H;R212H]ORF74 mutant (Figure 1a) has been shown previously to have high constitutive activity equivalent to the wild-type receptor (12). Here we show that similar to what was reported with human chemokines, this mutant responds to murine inverse agonists, but not the agonists. (Figure 1b). Finally, we tested a mutant ([L91D]ORF74) (Figure 1a), which has been deprived of its high constitutive activity (12). Murine and human agonists stimulated signal transduction, but the inverse agonists had no effect, in accordance with the fact that there is no constitutive activity to suppress (Figure 1b). Taken together, these results indicate that the wild-type ORF74 receptor and a

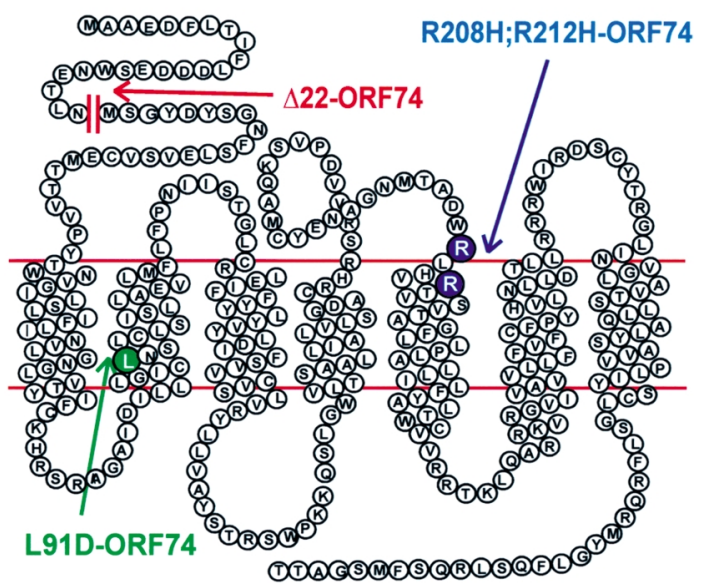

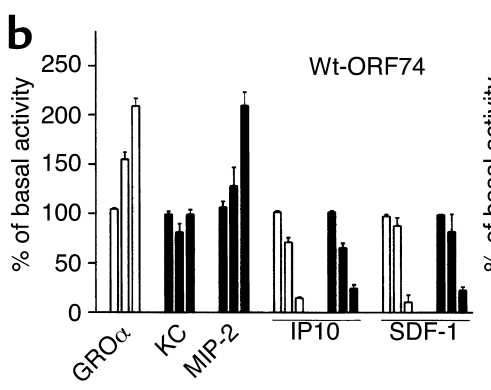
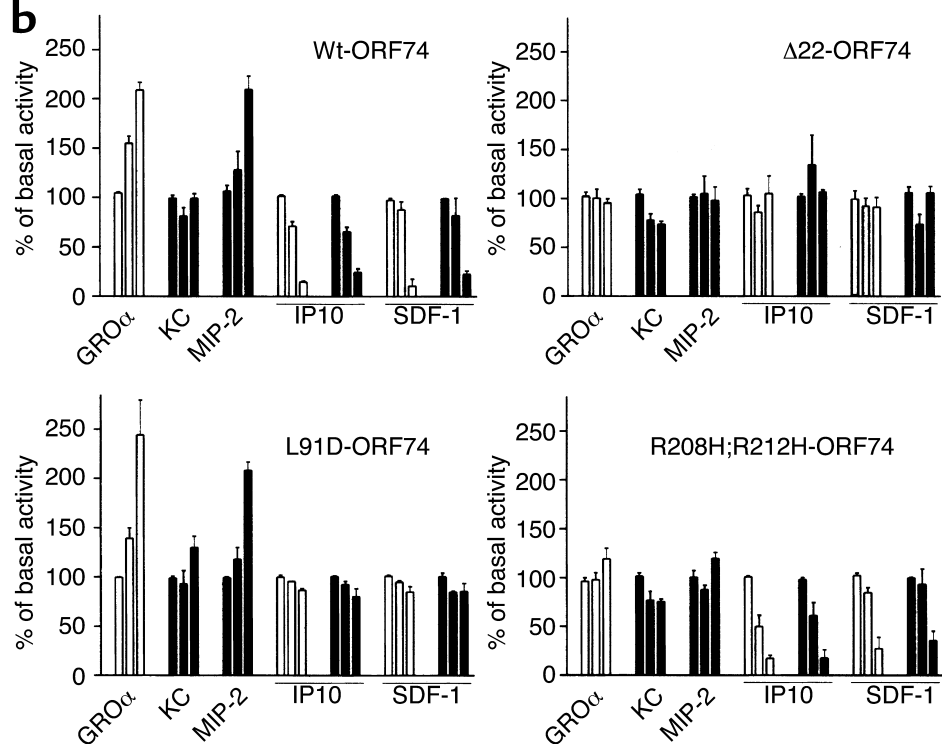

\section{Figure 1}

Regulation of ORF74 activity by human and murine CXC chemokines. (a) Diagram of ORF74 and its mutant forms. (b) Phosphatidyl inositol turnover in transiently transfected COS-7 cells stimulated by human (open columns) or murine chemokines (filled columns). Values are given in percentage of specific basal activity. Three columns are shown for each peptide corresponding to 0 , 1, and $100 \mathrm{nM}$ of peptide (from left to right). WT-ORF74: Full, potent activation by the human agonist GRO $\alpha$ as well as the murine homologue MIP-2, but not by KC, and potent, efficient inhibition by both the murine and human IP-10 and SDF-1 $\alpha$. $\Delta 22-O R F 74$ : No significant effect of any murine or human chemokine on the high constitutive signaling. L91D-ORF74: This mutant is fully and potently stimulated by both human GRO $\alpha$ and the murine MIP2, but there is no effect of either human or murine inverse agonists. R208H;R212H-ORF74: No stimulation by either murine or human agonists, but inhibition of constitutive activity by both murine and human inverse agonists. 


\begin{tabular}{|c|c|c|c|}
\hline$C D V G$ & $\mathrm{CD} 2$ & [WT]ORF74 & LCR \\
\hline L91D & CD2 & [L91D]ORF74 & LCR \\
\hline$\Delta 22$ & $\mathrm{CD} 2$ & [A22]ORF74 & LCR \\
\hline $\mathrm{R} 2 \mathrm{H}$ & CD2 & {$[\mathrm{R} 208 \mathrm{H} ; \mathrm{R} 212 \mathrm{H}] \mathrm{ORF} 74$} & LCR \\
\hline
\end{tabular}

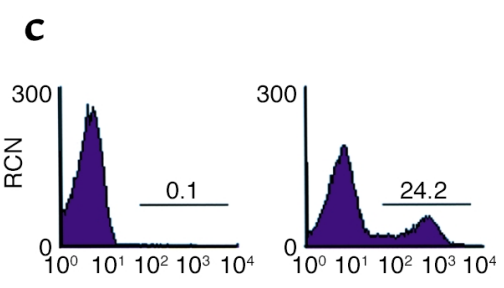

d

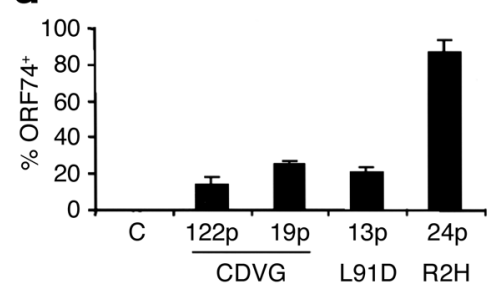

\section{Figure 2}

Generation and expression analysis of ORF74 transgenic mice. (a) Schematic description of the transgenes CDVG, L91D, $\Delta 22$, and R2H. Mutant forms of ORF74 described in Figure 1 were cloned into a vector containing the hCD2 promoter and locus control region (LCR). (b) Northern blot analysis of thymic RNA from control and transgenic mice. Shown are representative samples from CDVG (line 19), L91D (lines 15 and 13), $\Delta 22$ (lines 7 and 58), and R2H (line 24) animals. Lower panel shows the ethidium bromide-stained RNA gels. (c) Histogram of ORF74 expression on thymic cells from control and CDVG (line 19) transgenic mice. Transgene expression is distinct and easily detectable at the cell surface by flow cytometry. (d) Relative number of thymocytes expressing ORF74, estimated by flow cytometry, in CDVG (lines 19 and 122), L91D (line 13$)$, and $\mathrm{R} 2 \mathrm{H}$ (line 24). Bars represent average $\pm \mathrm{SD}(n=3)$. p, progeny. transgenic lines were obtained from six founders. Strikingly, all animals in three lines expressing ORF74 showed multiple angiogenic lesions within 90 days of life (Table 1). As described previously, these lesions were located in the ears, the tail, the nose, or the paws, and consisted initially of bright, sharply bordered erythematous plaques, which eventually progressed to nodules and subsequently to tumors (Figure $3 \mathrm{~d}$ ). Tumors were common after 150-200 days of life and were often found in the structures described above as well as in muscles of the lumbar region.

To test if the constitutive activity of ORF74 was essential for tumorigenesis we studied transgenic mice expressing the mutant L91D-ORF74. The basal activity of this receptor (as measured by PI turnover) in vitro is $18.9 \%$ of that detected for WT-ORF74, which is essentially similar to that found in cells transfected with an empty vector (12). Thirty-six independent transgenic founders were created carrying the L91D transgene. Twenty-five of these founders were aged to 200 days and euthanized. At necropsy there were no signs of angiogenic lesions or tumors, despite receptor expression in 10 of the

its mutant versions respond equivalently to human chemokines and their murine orthologues.

Generation of transgenic mice expressing ORF74 mutants. A number of founder transgenic mice expressing each of the ORF74 mutants described above were generated. The transgenes were driven by the hCD2 promoter, as described previously (7) and are referred to as CDVG ([WT]ORF74), L91D ([L91D]ORF74 mutant), $\Delta 22$ ([ $\Delta 22]$ ORF74 mutant), and, finally, R2H ([R208H;R212H]ORF74 mutant) (Figure 2a). Thirty-six transgenic founders were generated carrying the L91D transgene; eleven founders carrying the $\Delta 22$ transgene, and nine founders carrying the $\mathrm{R} 2 \mathrm{H}$ transgene. Founders were monitored daily for development of phenotypes and mated to generate transgenic lines. Seven lines were derived from L91D founders, seven lines from $\Delta 22$ founders, and eight lines from $\mathrm{R} 2 \mathrm{H}$ founders. Expression of the different transgenes was assessed by Northern blot analysis of thymic RNA or by flow cytometric analysis of thymocytes using a mAb against ORF74. A representative analysis of expression of the different transgenes is shown in Figure 2 (b-d).

Elimination of the constitutive signaling activity of ORF74 dramatically reduces disease incidence in transgenic mice. As reported previously, seven founders carrying the wild-type ORF74-encoded receptor were generated from pronuclear injections (7). Five independent
20 founders examined (data not shown). Next we examined receptor expression in seven lines derived from the L91D founders. Cell surface expression of the mutated receptor could be detected in animals in four of these lines (lines 13,15, 18, and 20; Figure 2, b and d), but no angiogenic lesions could be observed in ears, tails, or limbs by 90 days of age (Table 1). The incidence of angioproliferative disease in these animals was significantly different from that observed in the CDVG mice $(P<0.00036)$. Taken together, these results indicate that the constitutive activity of ORF74 is an essential element in the pathogenesis of the KS-like disease in transgenic mice.

Deletion of the chemokine-binding domain of ORF74 prevents the development of KS-like disease. To determine the importance of the chemokine-binding domain in the development of KS-like disease we studied transgenic mice bearing the [ $\Delta 22]$ ORF74 mutant (Figure 1a). This mutant is unable to respond to both human $(12,15)$ and mouse chemokines (Figure 1b), but preserves high constitutive activity (basal activity is $90 \%$ of that observed for WT-ORF74) (12). Eleven $\Delta 22$ founders were generated. All these founders survived past 90 days of age and five past 400 days. Strikingly, none of these mice developed angiogenic lesions or tumors. All the founders were mated, and eleven lines were generated. Seven out of the eleven $\Delta 22$ lines expressed the transgene by RNA analysis of thymic RNA (Figure 2b). 
Table 1

Incidence of KS-like disease in ORF74 transgenic mice

$\begin{array}{lc}\text { Founders } & \text { KS-like disease } \\ \text { CDVG } & 3 / 6(50 \%) \\ \Delta 22 & 0 / 11(0 \%) \\ \text { R2H } & 2 / 9(22 \%) \\ \text { L91D } & 0 / 25(0 \%)\end{array}$

\begin{tabular}{|c|c|c|c|c|c|c|c|}
\hline \multicolumn{8}{|c|}{ Lines } \\
\hline CDVG & KS-like disease & $\Delta 22$ & KS-like disease & $\mathrm{R} 2 \mathrm{H}$ & KS-like disease & L91D & KS-like disease \\
\hline 19 & $210 / 210(100 \%)$ & 7 & $0 / 13(0 \%)$ & 24 & $22 / 79$ (28\%] & 13 & $0 / 50(0 \%)$ \\
\hline 9 & $41 / 41(100 \%)$ & 53 & $0 / 5(0 \%)$ & 3 & $0 / 15(0 \%)$ & 15 & $0 / 10(0 \%)$ \\
\hline 122 & $39 / 39(100 \%)$ & 1 & $0 / 2(0 \%)$ & 26 & $0 / 8(0 \%)$ & 18 & $0 / 1(0 \%)$ \\
\hline 7 & $0 / 22(0 \%)$ & 58 & $0 / 2(0 \%)$ & 1 & $1 / 5(20 \%)$ & 20 & $0 / 1(0 \%)$ \\
\hline \multirow[t]{4}{*}{18} & $0 / 3(0 \%)$ & 60 & $0 / 1(0 \%)$ & 4 & $0 / 1(0 \%)$ & & \\
\hline & & 61 & $0 / 1(0 \%)$ & 15 & $0 / 2(0 \%)$ & & \\
\hline & & 66 & $0 / 1(0 \%)$ & 16 & $0 / 5(0 \%)$ & & \\
\hline & & 28 & $0 / 1(0 \%)$ & & & & \\
\hline
\end{tabular}

Animals were examined at 90 days of age for development of angiogenic lesions in the ears or limbs. Number of animals presenting lesions, number of total animals examined, and penetrance of the phenotype are indicated.

When examined at 90 days of age, none of the animals in these lines displayed angiogenic lesions (Table 1). Thus, within the first 3 months of life there was a significant $(P<0.0031)$ difference in the incidence of angiogenic disease between $\triangle 22$ and CDVG animals. Only one animal from line 58 developed a tumor on the tail at 300 days of age. The absence of angioproliferative lesions in the $\Delta 22$ mice suggests that chemokine binding to ORF74 is necessary for development of KS-like disease in transgenic mice.

Selective ablation of agonist-induced stimulation reduces the tumorigenic potential of ORF74. Having demonstrated that both the constitutive activity and the chemokinebinding domain of ORF74 are necessary for the development of KS-like disease in mice, we focused next on the following question: To what degree is the regulation of ORF74 activity important for the development of disease? As yet, we have been unable to generate a mutant in which inverse agonist function is selectively eliminated; but we have identified a mutant

\section{Figure 3}

Angiogenic and inflammatory lesions in CDVG, R2H and $\Delta 22$ transgenic mice. (a) Angiogenic lesions in the ear of a CDVG (line 19) mouse. (b) Angiogenic lesions in the ear of a R2H (line 24) mouse. (c) Erythematous, swollen, ulcerated ear lesions in a $\Delta 22$ (line 1 ) mouse. (d) Tail tumor in a CDVG (line 19) mouse. (e) Tail tumor in a $\mathrm{R} 2 \mathrm{H}$ line 24 mouse. (f) Ulcerations and scales in the tail of a $\Delta 22$ (line 1) animal. (g) H\&E-stained section of the tumor from the CDVG mouse shown in $\mathbf{d}$. The tumor is moderately pleomorphic, and many spindle-shaped cells arranged in interlacing fascicles are present. Several slitlike vascular spaces often containing erythrocytes are observed. (h) H\&E-stained section of the tumor from the $\mathrm{R} 2 \mathrm{H}$ mouse shown in e. The cellular composition and the arrangement of spindle cells in fascicles is indistinguishable from that found in CDVG mice (e). (i) H\&E-stained section of the ear of the $\Delta 22$ animal shown in c. The cellular infiltrate in inflamed ears consists mostly of granulocytes (eosinophils and neutrophils) and mononuclear cells.
([R208H;R212H]ORF74) (Figure 1a) that binds several murine chemokine ligands, has high constitutive activity (basal activity at $89 \%$ of WT-ORF74), but responds very poorly to agonist stimulation (12). Nine transgenic founder animals carrying this transgene $(\mathrm{R} 2 \mathrm{H})$ were generated, and eight expressing lines were derived (Table 1). Transgene expression was ascertained by both RNA analysis and flow cytometry (Figure $2, \mathrm{~b}$ and d). Only two of the nine founders devel-
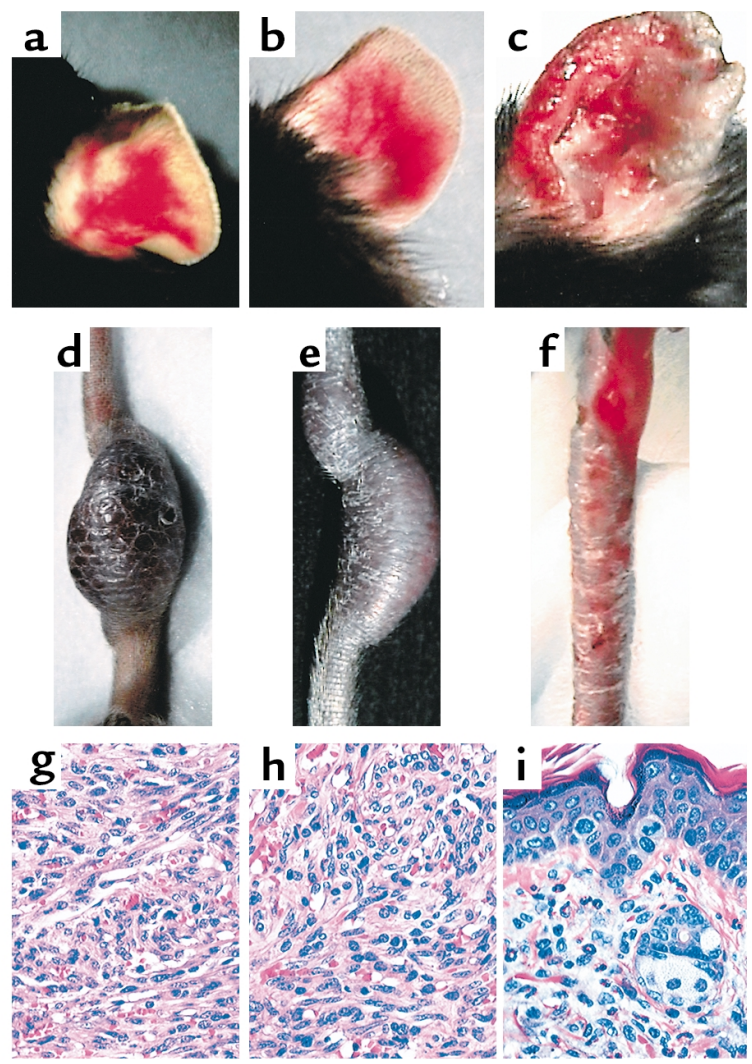


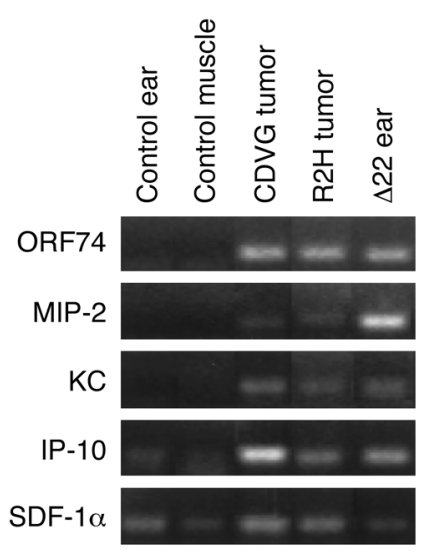

\section{Figure 4}

Expression of ORF74 and chemokines within lesions of transgenic mice. RT-PCR analysis of distinct CXC chemokines and ORF74 in control and transgenic tissues. ORF74 expression is detected in all transgenic samples, but not in control tissue. Expression of the ELR-chemokines KC and MIP-2 is detected within tumors of CDVG and R2H mice and in inflammatory lesions of $\Delta 22$ transgenic mice. The non-ELR chemokines SDF- $1 \alpha$ and IP-10 are expressed in both transgenic and control samples.

oped KS-like disease. One founder (no. 23) had an ear plaque that did not progress to nodules or tumors and the other (no. 15) had an erythematous lesion that progressed to a tail tumor after 150 days. Only two of the eight R2H lines (no. 1 and no. 24) developed a KS-like disease, and within these lines the penetrance of the phenotype was low (20\% and $28 \%$, respectively) (Table 1). These angiogenic lesions (and tumors) were histologically similar to those observed in mice expressing the wild-type receptor (Figure 3, b, e, and h). Interestingly, not only did a smaller fraction of mice within the affected lines develop KS-like disease, but also the severity of the disease was reduced when compared with that seen in the mice expressing the wildtype receptor. Taken together, the incidence of angiogenic disease in $\mathrm{R} 2 \mathrm{H}$ founders and lines was significantly lower than that observed in the CDVG founders and lines $(P<0.036)$. Based on these results, we conclude that the mutations affecting the ability of ORF74 to respond to agonists such as MIP-2 (and possibly other murine chemokines) reduce its ability to induce angiogenic lesions and tumors in vivo.

The ORF74 chemokine ligands are expressed within the KSlike lesions. The absence of KS-like disease in the $\Delta 22$ mice and the reduced penetrance of angiogenic phenotype in $\mathrm{R} 2 \mathrm{H}$ mice at 90 days of age were highly suggestive of a regulatory role for endogenous chemokines in the development of disease. We therefore examined the expression of murine chemokine ligands within lesional tissue of transgenic animals. As shown in Figure 4, the CXC-ELR chemokines KC and MIP-2 were both detected within angiogenic lesions of most samples examined, but not in the control samples. In contrast, the non-ELR CXC chemokines IP-10 and SDF- $1 \alpha$ were expressed in most lesions and in the control samples. These results confirmed expression of different forms of ORF74 and its ligands within lesional tissue and raise the possibility of local modulation by endogenous chemokines of receptor activity. Inflammatory phenotypes are observed in CDVG, 422 , $L 91 D$, and R2H mice. Inflammatory lesions affecting skin and, to a lesser extent, the joints and the intestinal tract, were apparent in some of the transgenic mice but never in wild-type littermates. Such lesions were observed in one line of mice expressing the wild-type ORF74 receptor, in all seven $\Delta 22$ lines with varying penetrance, and in five out of the eight lines expressing the $\mathrm{R} 2 \mathrm{H}$ transgene. These lesions were not detected in any of the L91D founders, but were seen in approximately $20 \%$ of animals of one of the four L91D lines (no. 13) after 120 days of age. In most cases the skin lesions ulcerated spontaneously, giving rise to scabs on the tail, ears, and face (Figure 3, c and f). Histologically, the skin lesions were characterized by hyperkeratosis, acanthosis, and inflammatory infiltrates rich in both polymorphonuclear and mononuclear cells (Figure 3i). Swollen joints and rectal prolapse were more rarely observed.

\section{Discussion}

KS is an angioproliferative disease epidemiologically linked to HHV8 $(2,16,17)$. Among the many genes encoded by HHV8 is ORF74, a constitutively highlyactive chemokine receptor that binds a number of CXC chemokines (10). Surprisingly, expression of ORF74 is sufficient to induce a KS-like disease in transgenic mice, suggesting a central role for this receptor in the pathogenesis of KS (7). In this study we investigated the mechanisms associated with the induction of the angioproliferative disease, focusing on the constitutive versus regulated signaling properties of ORF74.

Experiments carried out in vitro have suggested that the constitutive signaling activity of ORF74 is a pivotal element in the induction of $\mathrm{KS}(5,6,9)$. To examine the contribution of the constitutive activity of ORF74 to the development of KS-like disease we generated several founders and transgenic lines carrying a mutated form of ORF74 (L91D-ORF74) capable of ligand binding and activation, but deficient in constitutive signaling. Despite expression of the mutant receptor at the cell surface level, none of the transgenic mice developed angiogenic lesions by 90 days of age. These results confirm that the constitutive signaling activity of ORF74 is required for the development of KS-like disease.

ORF74 has been optimized by HHV8 not only for high constitutive signaling, but also for high-affinity binding of a number of endogenous chemokines of its host. Interestingly, the chemokines that act as agonists on ORF74, i.e., further increase the signaling activity, are all angiogenic chemokines, with GRO $\alpha$ being the most potent example $(10,11,15,18)$. Moreover, the two chemokines (IP-10 and SDF-1 $\alpha$ ) that act as inverse agonists, i.e., decrease the signaling toward basal level, function as angiostatic chemokines in vivo $(9,10,18)$. Thus, both positive and negative modulation of the high constitutive signaling activity of ORF74 by 
endogenous chemokines could be important for the development of the highly vascularized lesions. This hypothesis is supported by several observations reported here. First, deletion of the far N-terminal segment of ORF74, which deprives the receptor of its ability to bind any chemokine, prevented the formation of the KS-like tumors in the transgenic animals, despite the fact that the mutant receptor still displayed unaltered high constitutive signaling activity. Second, expression of the mutant [R208H;R212H]ORF74, which preserved high constitutive activity but failed to respond to the agonist chemokines GRO $\alpha$ and its murine orthologue MIP-2, was associated with reduced penetrance and severity of the KS-like disease. Third, expression of the angiogenic and angiostatic chemokines, which have been shown to modulate ORF74 activity, was detected within the lesional tissue of the transgenic mice. Taken together, these results indicate that modulation of the high constitutive activity of ORF74 by endogenous chemokines is an important factor in the development of a KS-like disease in mice.

Expression of ORF74 and its mutant forms in transgenic mice was also associated with development of inflammatory conditions affecting predominantly the skin and, to a lesser extent, the joints and gastrointestinal tract. These conditions may have been caused by cells expressing these receptors within the inflammatory sites. Alternatively, they may have resulted from expression of the receptor(s) within thymic cells. This hypothesis is based on our recent observations that thymic development is affected in many of the transgenic mice reported here (D. Manfra, unpublished observations). Altered thymic development may have led to a systemic immune dysfunction favoring the development of the inflammatory conditions, as documented in several other models (19-21). Further studies are underway to define the mechanistic basis of these inflammatory phenotypes. Results presented here point to co-optation and optimization by the HHV8 of basic signal transduction pathways triggered by chemokine receptors, in particular those implicated in angiogenesis. Several studies in the literature have implicated chemokines of the CXCfamily in angiogenesis, angiostasis, tumor growth, and tumor regression (for review see ref. 18). The CXCchemokines containing the ELR-motif have been shown to be angiogenic factors in vitro and in vivo and to be capable of enhancing tumor growth of immortalized cells, whereas the ELR-lacking CXC-chemokines have been shown to have opposite effects in vivo $(11,18$, 22-25). Here we find that modulation or fine-tuning of the signaling activity of ORF74 by endogenous angiogenic chemokines is required for the development of highly vascularized tumors. ORF74 may thus represent a molecular switch to initiate a program of gene expression that will affect multiple functions, including angiogenesis. One of the genes affected by ORF74 expression is the potent angiogenic factor VEGF, whose expression has been demonstrated in endothelial and fibroblast cells transfected with ORF74, within KS lesions, and within ORF74-induced tumors in transgenic mice (5, 7, 26). Other likely candidates would be the proinflammatory cytokine IL- $1 \beta$ and basic fibroblast growth factor. These molecules, which are also frequently detected within KS lesions, are induced in cells transfected with ORF74 $(4,27)$. Because we cannot formally assume that ORF74 signaling will result in activation of the same genes in transfected cells and in transgenic cells, it will be important to examine the expression of the genes downstream of ORF74 in vivo. These studies will certainly contribute to characterization of the molecules involved in the angioproliferative disease described here.

Expression of chemokines by human tumor cells and tumor-infiltrating leukocytes has been amply documented in the literature, but it is unclear why they should be produced under these circumstances. Several potential roles have been proposed, including direct control of cellular proliferation, control of angiogenesis, and regulation of macrophage and dendritic cell function (28). Here we present evidence to suggest a novel role for chemokines in tumor biology, namely, regulation (by CXC chemokines) of the activity of a chemokine receptor oncogene. This virally encoded chemokine receptor acts as a nonconventional oncogene to promote production of paracrine factors that drive proliferation of endothelial cells and recruitment of inflammatory cells $(7,29)$. It is unclear whether local chemokines, besides affecting ORF74 activity, affect angiogenesis through interaction with other receptors, such as CXCR2, CXCR3, and CXCR4. These chemokine receptors are expressed in the vascular endothelium and have been implicated in angiogenesis (30-33). In this context, it will be of interest to investigate if chemokines and their receptors influence angiogenesis in normal and tumor settings, primarily through paracrine mechanisms.

In summary, the results described here implicate chemokines in the development of KS-like lesions in transgenic mice. We propose that modulation of ORF74 activity by chemokines may also represent a key step in the generation of KS in humans and suggest that the model described here represents the first example of a chemokine-driven neoplastic condition.

\section{Acknowledgments}

We thank P. Zalamea for technical assistance; D. Kioussis, E. Kremmer, and Steffen Posner for reagents; J. Hilden for statistical analysis; and J. Hedrick and W. Hipkin for comments on the manuscript. The study was supported in part by grants from the Biotechnology competence center for 7TM receptors and the Danish Cancer Association.

\footnotetext{
1. Chang, Y., et al. 1994. Identification of herpesvirus-like DNA sequences in AIDS-associated Kaposi's sarcoma. Science. 266:1865-1869.

2. Gao, S.J., et al. 1996. Seroconversion to antibodies against Kaposi's sarcoma-associated herpesvirus-related latent nuclear antigens before the development of Kaposi's sarcoma. N. Engl. J. Med. 335:233-241.

3. Staskus, K.A., et al. 1997. Kaposi's sarcoma-associated herpesvirus gene expression in endothelial (spindle) tumor cells. J. Virol. 71:715-719.

4. Antman, K., and Chang, Y. 2000. Kaposi's sarcoma. N. Engl. J. Med. 342:1027-1038.

5. Bais, C., et al. 1998. G-protein-coupled receptor of Kaposi's sarcoma-
} 
associated herpesvirus is a viral oncogene and angiogenesis activator [erratum 1998, 392:210]. Nature. 391:86-89.

6. Arvanitakis, L., Geras-Raaka, E., Varma, A., Gershengorn, M.C., and Cesarman, E. 1997. Human herpesvirus KSHV encodes a constitutively active G-protein- coupled receptor linked to cell proliferation. Nature. 385:347-350.

7. Yang, T.Y., et al. 2000. Transgenic expression of the chemokine receptor encoded by human herpesvirus 8 induces an angioproliferative disease resembling Kaposi's sarcoma. J. Exp. Med. 191:445-454.

8. Geras-Raaka, E., et al. 1998. Inhibition of constitutive signaling of Kaposi's sarcoma-associated herpesvirus $\mathrm{G}$ protein-coupled receptor by protein kinases in mammalian cells in culture. J. Exp. Med. 187:801-806.

9. Geras-Raaka, E.,Varma, A., Ho, H., Clark-Lewis, I., and Gershengorn, M.C. 1998. Human interferon-gamma-inducible protein 10 (IP-10) inhibits constitutive signaling of Kaposi's sarcoma-associated herpesvirus G protein-coupled receptor. J. Exp. Med. 188:405-408.

10. Rosenkilde, M.M., Kledal, T.N., Brauner-Osborne, H., and Schwartz, T.W. 1999. Agonists and inverse agonists for the herpesvirus 8-encoded constitutively active seven-transmembrane oncogene product, ORF-74. J. Biol. Chem. 274:956-961.

11. Strieter, R.M., et al. 1995. The functional role of the ELR motif in CXC chemokine-mediated angiogenesis. J. Biol. Chem. 270:27348-27357.

12. Rosenkilde, M.M., Kledal, T.N., Holst, P.J., and Schwartz, T.W. 2000 Selective elimination of high constitutive activity or chemokine binding in the human herpesvirus 8 encoded seven transmembrane oncogene ORF74. J. Biol. Chem. 275:26309-26315.

13. Berridge, M.J., Dawson, R.M., Downes, C.P., Heslop, J.P., and Irvine, R.F. 1983. Changes in the levels of inositol phosphates after agonist-dependent hydrolysis of membrane phosphoinositides. Biochem. J. 212:473-482.

14. Lipp, M., et al. 2000. Functional organization of secondary lymphoid organs by the chemokine system. Curr. Top. Microbiol. Immunol. 251:173-179.

15. Gershengorn, M.C., Geras-Raaka, E., Varma, A., and Clark-Lewis, I. 1998. Chemokines activate Kaposi's sarcoma-associated herpesvirus $\mathrm{G}$ protein-coupled receptor in mammalian cells in culture. J. Clin. Invest. 102:1469-1472.

16. Gao, S.J., et al. 1996. KSHV antibodies among Americans, Italians and Ugandans with and without Kaposi's sarcoma. Nat. Med. 2:925-928.

17. Kedes, D.H., et al. 1996. The seroepidemiology of human herpesvirus 8 (Kaposi's sarcoma-associated herpesvirus): distribution of infection in KS risk groups and evidence for sexual transmission. Nat. Med. 2:918-924.

18. Belperio, J.A., et al. 2000. CXC chemokines in angiogenesis. J. Leukoc. Biol. 68:1-8.
19. Ludviksson, B.R., Gray, B., Strober, W., and Ehrhardt, R.O. 1997. Dysregulated intrathymic development in the IL-2-deficient mouse leads to colitis-inducing thymocytes. J. Immunol. 158:104-111.

20. Kretz-Rommel, A., and Rubin, R.L. 2000. Disruption of positive selection of thymocytes causes autoimmunity. Nat. Med. 6:298-305.

21. Seddon, B., and Mason, D. 2000. The third function of the thymus. Immunol. Today. 21:95-99.

22. Angiolillo, A.L., et al. 1995. Human interferon-inducible protein 10 is a potent inhibitor of angiogenesis in vivo. J. Exp. Med. 182:155-162.

23. Sgadari, C., et al. 1996. Interferon-inducible protein-10 identified as a mediator of tumor necrosis in vivo. Proc. Natl. Acad. Sci. USA 93:13791-13796

24. Fujisawa, N., Hayashi, S., and Miller, E.J. 1999. A synthetic peptide inhibitor for alpha-chemokines inhibits the tumour growth and pulmonary metastasis of human melanoma cells in nude mice. Melanoma. Res. 9:105-114.

25. Fujisawa, N., Hayashi, S., Kurdowska, A., Carr, F.K., and Miller, E.J. 1999. Inhibition of GROalpha-induced human endothelial cell proliferation by the alpha-chemokine inhibitor antileukinate. Cytokine. 11:231-238.

26. Sodhi, A., et al. 2000. The Kaposi's sarcoma-associated herpes virus $G$ protein-coupled receptor up-regulates vascular endothelial growth factor expression and secretion through mitogen-activated protein kinase and $\mathrm{p} 38$ pathways acting on hypoxia-inducible factor 1alpha. Cancer Res. 60:4873-4880.

27. Schwarz, M., and Murphy, P.M. 2001. Kaposi's sarcoma-associated herpesvirus $\mathrm{G}$ protein-coupled receptor constitutively activates Nf-kappaB and induces proinflammatory cytokine and chemokine production via a C-terminal signaling determinant. J. Immunol. 167:505-513.

28. Rossi, D., and Zlotnik, A. 2000. The biology of chemokines and their receptors. Annu. Rev. Immunol. 18:217-242.

29. Gruber, B.L., Marchese, M.J., and Kew, R. 1995. Angiogenic factors stimulate mast-cell migration. Blood. 86:2488-2493.

30. Vicari, A.P., et al. 2000. Antitumor effects of the mouse chemokine 6Ckine/SLC through angiostatic and immunological mechanisms. J. Immunol. 165:1992-2000.

31. Addison, C.L., et al. 2000. The CXC chemokine receptor 2, CXCR2, is the putative receptor for $\mathrm{ELR}(+) \mathrm{CXC}$ chemokine-induced angiogenic activity. J. Immunol. 165:5269-5277.

32. Murdoch, C., Monk, P.N., and Finn, A. 1999. Cxc chemokine receptor expression on human endothelial cells. Cytokine. 11:704-712.

33. Rempel, S.A., Dudas, S., Ge, S., and Gutierrez, J.A. 2000. Identification and localization of the cytokine SDF1 and its receptor, CXC chemokine receptor 4 , to regions of necrosis and angiogenesis in human glioblastoma. Clin. Cancer Res. 6:102-111. 